\title{
Impurity Segregation via Extended Defects in Oxide Thin Films Probed by Aberration-Corrected STEM-EELS
}

\author{
David J. Baek ${ }^{1}$, Di Lu ${ }^{2}$, Yasuyuki Hikita ${ }^{3}$, Harold Y. Hwang ${ }^{3,4}$ and Lena F. Kourkoutis ${ }^{5,6}$ \\ 1. School of Electrical and Computer Engineering, Cornell University, Ithaca, NY, USA. \\ 2. Department of Physics, Stanford University, Stanford, CA, USA. \\ 3. Stanford Institute for Materials and Energy Sciences, SLAC National Accelerator Laboratory, Menlo \\ Park, CA, USA. \\ 4. Department of Applied Physics, Stanford University, Stanford, CA, USA. \\ 5. School of Applied and Engineering Physics, Cornell University, Ithaca, NY, USA. \\ ${ }^{6 .}$ Kavli Institute at Cornell for Nanoscale Science, Cornell University, Ithaca, NY, USA.
}

To realize novel functional properties in perovskites, oxide thin films are often deposited on a dissimilar underlying substrate. However, as a consequence, misfit dislocations (MD) can form at the interface as a strain relaxation mechanism during growth. This in turn allows stress-assisted segregation of impurities to occur at the defects. As physical properties of oxides depend heavily on stoichiometric growth, it is critical to understand the precise role defects play during impurity segregation and migration. To address this issue, here, we employ aberration-corrected STEM-EELS to obtain atomic-scale information of the structural, chemical, and electronic changes that take place around defects. The oxide system probed in this work was prepared by depositing a $\left[\mathrm{La}_{0.7} \mathrm{Sr}_{0.3} \mathrm{MnO}_{3}\right]_{5} /\left[\mathrm{SrTiO}_{3}\right]_{5}$ superlattice (SL) film on top of the $\mathrm{SrTiO}_{3}(001)$ substrate with an intermediate layer of the elastic perovskite-like material $\mathrm{Sr}_{3} \mathrm{Al}_{2} \mathrm{O}_{6}(\mathrm{SAO})$ using pulsed laser deposition [1].

Due to the lattice constant mismatch between the layers (LSMO: $3.88 \AA$, STO: $3.905 \AA$, SAO: $3.961 \AA$ ), MDs form at both SL/SAO and SAO/STO interfaces as shown in the HAADF-STEM image in Figure 1 (red boxes). More interestingly, extended defects (yellow arrows) spanning the SL film are observed and bridge directly into the MDs at the upper SAO interface. LAADF-STEM image further shows that the extended defects in the SL film can extend into the SAO layer and all the way to the lower SAO interface where additional MDs exist. While the visibility of such defects can be improved by using LAADF-STEM, variations in the local chemistry can be more easily extracted using HAADF-STEM. Compared to the dark contrast at MDs in HAADF-STEM images, extended defects in the SL film appear brighter than their surrounding suggesting the presence of heavier elements. To clarify the chemical identities of the atoms confined within the extended defects, EELS analysis was performed for Ti- $\mathrm{L}_{2,3}, \mathrm{Mn}-\mathrm{L}_{2,3}$, and La-M $\mathrm{M}_{4,5}$ edges. The spectroscopic image includes two extended defects as indicated by white arrows in the Ti map in Figure 1. While Ti shows negligible signal in the SAO film as expected, surprisingly, La and $\mathrm{Mn}$ are detected with increased concentration at the two interfaces where a high density of MDs exist. These results imply that Mn and La atoms diffuse through dislocation cores as previously reported for other oxide systems [2,3]. To explore possible electronic changes at the defects, multivariate curve resolution (MCR) was performed for the Mn-L edge. Two distinct Mn components are extracted, one with reduced Mn valence as reflected in the Mn-L edge fine structure. Mn atoms with the reduced valence state more strongly occupy the two interfaces and the lower part of the SL film where MDs and extended defects exist. Finally, to confirm the migration of impurities through the extended defects at the atomic-scale, higher resolution EELS was conducted. As shown in the elemental maps of Figure 2, La and Mn occupy atomic sites in the extended defects. Furthermore, Ti is deficient at the defect, which is consistent with Mn atoms filling these sites. A similar MCR analysis verifies that 
Mn atoms migrating via the extended defect exhibit a reduced valence state, which is consistent with the observation above.

[1] D Lu et al, MRS Spring (2014), K12.11.

[2] C. -P Chang et al, Nat. Commun. 5 (2014), p. 1.

[3] M Arredondo et al, Adv. Mater. 22 (2010), p. 2430.

[4] This work was supported by the Cornell Center for Materials Research with funding from the NSF MRSEC program (DMR-1120296) and the Department of Energy, Office of Basic Energy Sciences, Division of Materials Sciences and Engineering, under contract DE-AC02-76SF00515.

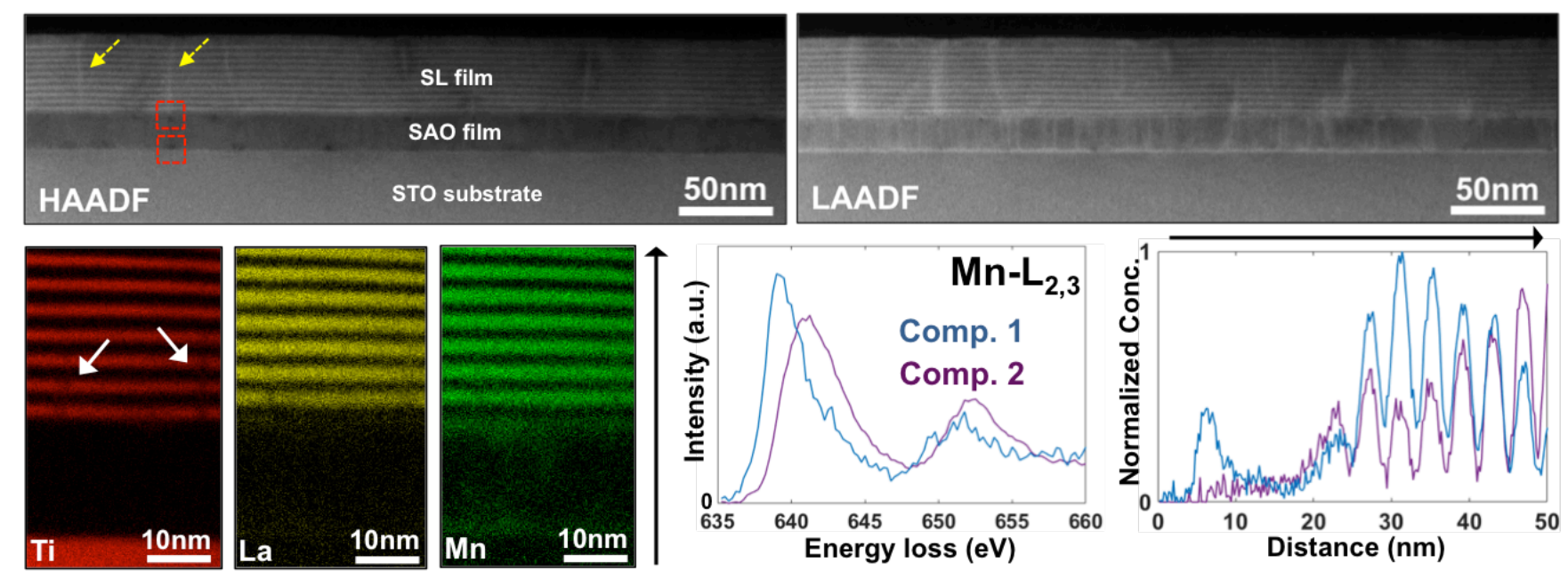

Figure 1. Upper figures: Lower magnification HAADF-STEM and LAADF-STEM images showing the overall structure of the material with the presence of crystal defects. Yellow arrows and red boxes indicate extended defects and misfit dislocations, respectively. Lower figures from left to right: EELS elemental maps of $\mathrm{Ti}, \mathrm{La}$, and $\mathrm{Mn}$. White arrows in Ti map indicate the presence of two extended defects. Two rightmost figures each show the Mn-L spectrum and the concentration profile of two distinct Mn components extracted by multivariate curve resolution (MCR). A reduced valence state is observed for Mn atoms that segregate at the defects.
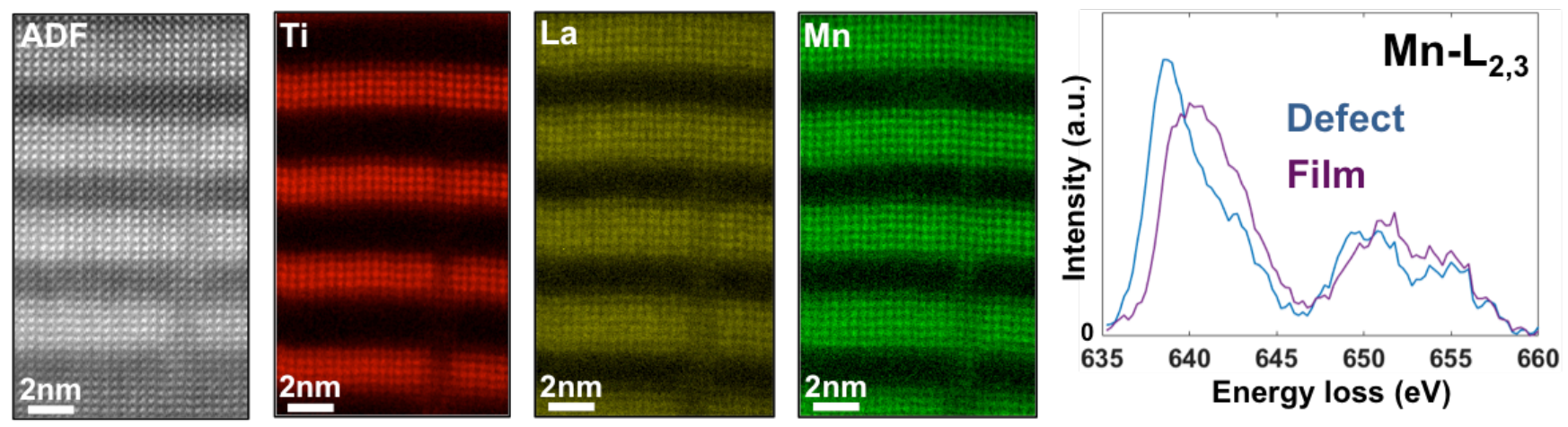

Figure 2. Left to right: Atomic-resolution ADF image followed by EELS elemental maps of Ti, La, and $\mathrm{Mn}$ acquired across an extended defect in the SL film. Similar analysis using MCR confirms that Mn atoms confined within the extended defect exhibit a reduced valence state consistent with the observed local chemistry. 